\title{
Noradrenergic Hyperinnervation in the Heart of Stroke-Prone Spontaneously Hypertensive Rats (SHRSP)
}

\author{
Mari Kondo, Takashi Fujiwara*, and Ryo Tabei
}

\begin{abstract}
Noradrenergic (NA) nerve fiber density was investigated in the subepicardium and myocardium of ventricles in stroke-prone spontaneously hypertensive rats (SHRSP) and was compared with that of normotensive Wistar Kyoto (WKY) rats. Fluorescent NA nerve fibers in the subepicardium of the right and left ventricles of both strains at the ages of $10,30,60,90$, and 180 days were examined by the glyoxylic acid method. NA nerve fibers in the myocardium of the right and left ventricles and the ventricular septum of both strains at the ages of 30,90, and 180 days were also examined in a similar manner. The density of NA nerve fibers was measured by quantitative image analysis. The distribution pattern of NA nerve fibers in the entire subepicardium of ventricles of both strains showed a meshwork pattern throughout the examination period. In sections of the myocardium, NA nerve fibers were distributed between heart muscle cells and around blood vessels in both strains at all ages examined. The densities of NA nerve fibers in the subepicardium of the ventricles of SHRSP were significantly higher than those of WKY rats at all ages examined except for the subepicardium of the left ventricle at 90 days of age. The densities in the myocardium of the right ventricle in 30- and 90-day-old SHRSP were significantly higher than those in WKY rats. The ratios of NA nerve fiber density of SHRSP to that of WKY rats were greater in the subepicardium of the right and left ventricles, except at 90 days of age, and in the myocardium of the right ventricle of younger animals as compared with older ones. NA hyperinnervation in the subepicardium and myocardium of the ventricles of SHRSP may be a primary change of the heart before the onset of hypertension and may be caused by hyperfunction of the stellate ganglia. (Hypertens Res 1996; 19: 69-73)
\end{abstract}

Key Words: noradrenergic nerve fiber, heart, hypertension, stroke-prone spontaneously hypertensive rat

The superior cervical and stellate ganglia of spontaneously hypertensive rats (SHR) have been reported to exhibit hyperfunction as compared with those of normotensive Wistar Kyoto (WKY) rats $(1-3)$. Fluorescence microscopic studies have shown that the densities of noradrenergic (NA) nerve fibers of the middle cerebral artery (4) and the ophthalmic artery (5), which originate from the superior cervical ganglia, were increased in strokeprone spontaneously hypertensive rats (SHRSP) as compared with those in WKY rats. The density of NA fibers of the coronary artery, which originate from the stellate ganglia, was also higher in SHRSP than in WKY rats (6), suggesting that the NA content in the heart of SHRSP is higher than that of WKY rats. It is, however, unclear whether the NA nerve fibers in the myocardium, which also originate from the stellate ganglia, are more dense in SHRSP than in WKY rats. In the present study, we used morphological techniques to determine whether the sympathetic innervation density of the heart of hypertensive rats is different from that of the heart of normotensive WKY rats.

\section{Materials and Methods}

All animals used in this study were maintained on a normal laboratory diet and tap water ad libitum at the Laboratory Animal Center of Ehime University School of Medicine.

The animals used to study the subepicardium of the ventricles were 10-, 30-, 60-, 90-, and 180-dayold and those to study the myocardium were 30 -, 90-, and 180-day-old male SHRSP and WKY rats.

The method of specimen preparation was similar to that previously reported (5). Six animals of each age and strain were anesthetized with pentobarbital sodium $(20 \mathrm{mg} / \mathrm{kg}$ body weight) and were then per-

From the Department of Pathology, Ehime University School of Medicine, Ehime, and Laboratory Animal Center, Ehime University School of Medicine, Ehime, Japan.

Address for Reprints: Mari Kondo, Department of Pathology, Ehime University School of Medicine, Shitsukawa, Shigenobu, Onsen-gun, Ehime 791-02, Japan.

Received March 24, 1995; accepted in revised form December 26, 1995. 
fused with phosphate-buffered saline followed by $2 \%$ glyoxylic acid in phosphate buffer solution. After the heart was divided into right and left ventricular walls, the epicardium was peeled off a central part of each ventricular wall. Subsequently, the epicardium was immersed in the same glyoxylic acid solution at room temperature for $40 \mathrm{~min}$. It was then immersed in a drop of glyoxylic acid solution on non-fluorescent glass slides, and the glyoxylic acid solution was removed with a piece of filter paper after the epicardiums of right and left ventricles were spread on the slide. On the other hand, the myocardium was divided into right, left, and septal portions. The central part of each tissue block of myocardium was cut into frozen sections, $20 \mu \mathrm{m}$ thick, with a cryostat. The sections were spread on non-fluorescent glass slides. These two kinds of preparations, the epicardium and myocardium of the ventricles, were dried at room temperature, $22^{\circ}$ to $26^{\circ} \mathrm{C}$, for $20 \mathrm{~min}$ and then heated at $100^{\circ} \mathrm{C}$ for 7 min and mounted with paraffin oil. Four to five fields per specimen were photographed at a magnification of $\times 250$ with a Zeiss photomicroscope.

The distribution densities of NA nerve fibers in each micrograph were evaluated by quantitative image analysis using an interactive Bildanalyse System image analyzer (Kontron Bildanalyse). The density was expressed as a percentage of the area occupied by fluorescent nerve fibers per whole area of epicardium and myocardium $(7,8)$.

Student's $t$ test was used to evaluate the statistical significance of differences between SHRSP and WKY rats.

\section{Results}

NA nerve fibers in the subepicardium and myocardium of ventricles exhibited the characteristic green fluorescence of noradrenaline (9). In the subepicardium of the ventricles, varicose NA nerve fibers showed a meshwork pattern at all ages examined (Fig. 1). The thickness of the nerve fiber bundles decreased after 30 days of age. The densities of NA nerve fibers in the subepicardium of the right and left ventricles from SHRSP reached a peak at 30 days of age, decreased rapidly from 30 to 60 days of age, and remained rather constant thereafter. In contrast, those of WKY rats did not change remarkably at any age examined. The nerve densities in the subepicardium of the right and left ventricles in SHRSP were significantly $(p<0.01$ and 0.05$)$ higher than those of age-matched WKY rats at all ages examined with the exception of the subepicardium of the left ventricle at 90 days of age (Fig. 2 and 3). The difference in the nerve densities between SHRSP and WKY rats was greatest at 10 days of age (1.5 times) and decreased thereafter (Table 1).

NA nerve fibers in the myocardium of ventricles ran between cardiac muscle cells and around blood vessels in both strains (Fig. 4). More fibers were observed near the epicardium than near the endocardium. The bundle thickness was unchanged at all ages examined. The nerve fiber density in most portions of the myocardium increased with age in both strains. Although there were no significant differences in the nerve fiber densities of the left ven-
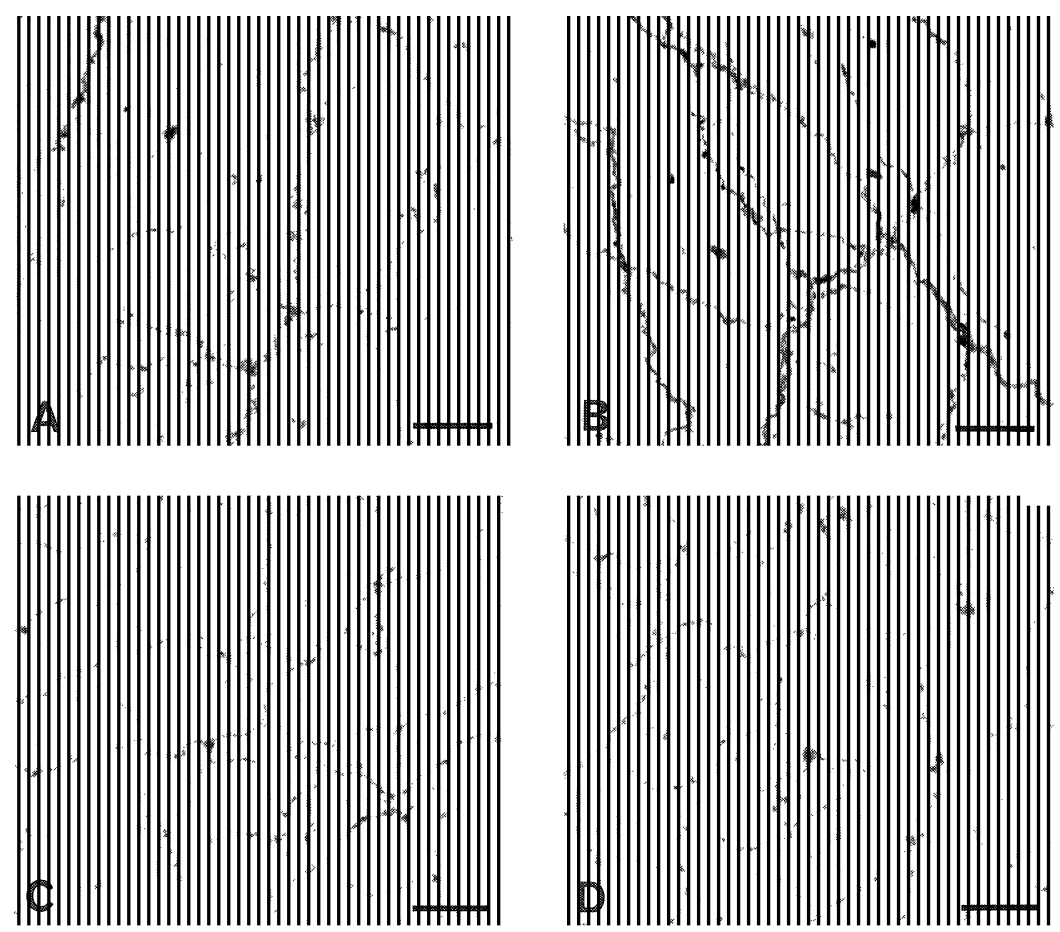

Fig. 1. Photomicrographs showing noradrenergic nerve fibers in the subepicardium of the right ventricle of $S H R S P(B, D)$ and $W K Y$ rats $(A, C)$ at 10 days $(A, B)$ and 30 days $(C, D)$ after birth. Bars $=20 \mu \mathrm{m}$. 


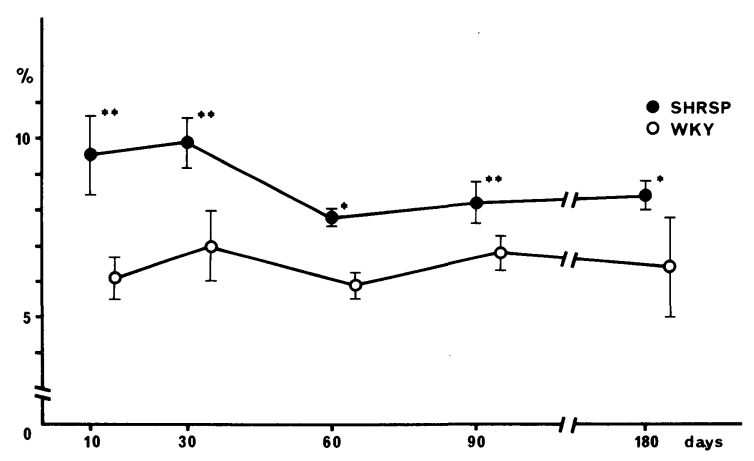

Fig. 2. Comparison of the density of noradrenergic nerve fibers in the subepicardium of the right ventricle between SHRSP and WKY rats at various ages. $* \mathrm{p}<0.05$ compared with $W K Y$ rats, ** $\mathrm{p}<0.01$ compared with WKY rats.

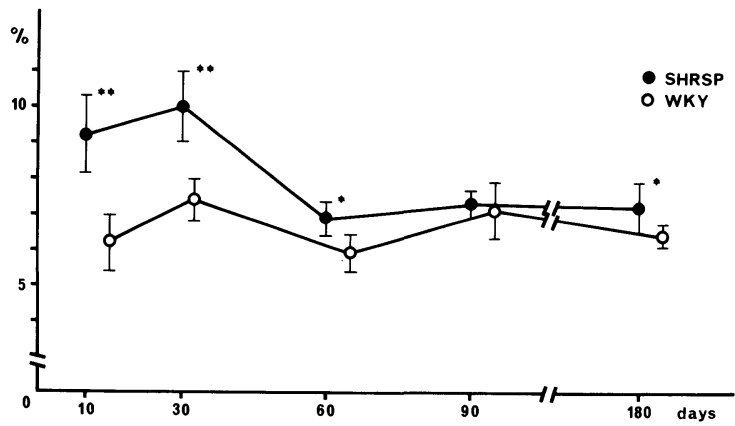

Fig. 3. Comparison of the density of noradrenergic nerve fibers in the subepicardium of the left ventricle between $S H R S P$ and $W K Y$ rats at various ages.

$* \mathrm{p}<0.05$ compared with $W K Y$ rats, ** $\mathrm{p}<0.01$ compared with WKY rats.

Table 1. The Ratios of Noradrenergic Nerve Density in the Heart of SHRSP to That of WKY Rats

\begin{tabular}{|c|c|c|c|c|c|}
\hline \multirow[t]{2}{*}{ Days after birth } & \multicolumn{2}{|c|}{ Subepicardium of ventricle } & \multicolumn{3}{|c|}{ Myocardium of ventricle } \\
\hline & Right & Left & Right & Left & Septum \\
\hline 10 & 1.5 & 1.5 & & & \\
\hline 30 & 1.4 & 1.4 & 1.5 & 1.0 & 1.0 \\
\hline 60 & 1.3 & 1.2 & & & \\
\hline 90 & 1.2 & 1.0 & 1.4 & 1.0 & 1.0 \\
\hline 180 & 1.3 & 1.1 & 1.1 & 1.0 & 1.0 \\
\hline
\end{tabular}

The figures are ratios obtained by dividing the value in SHRSP by that in WKY rats.
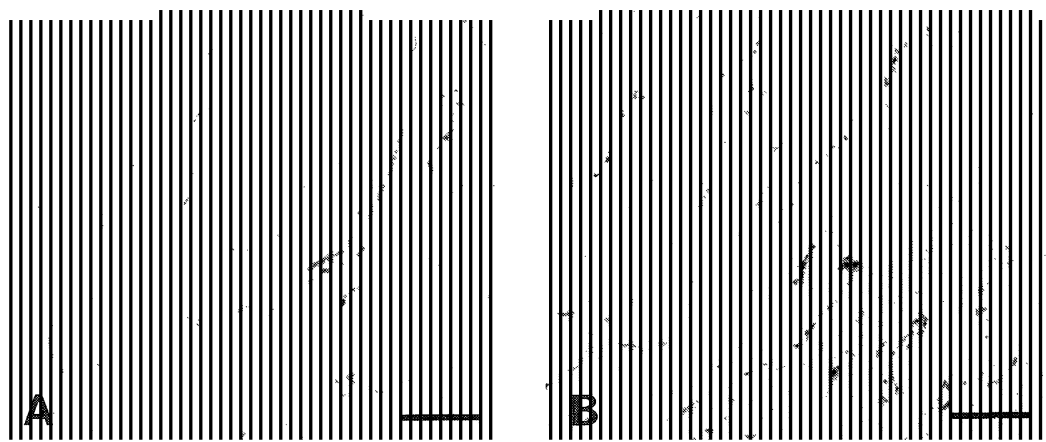

Fig. 4. Photomicrographs showing the noradrenergic nerve fibers in the myocardium of the right ventricle of SHRSP (B) and $W K Y$ rats $(A) 30$ days after birth.

tricle and the ventricular septum between SHRSP and WKY rats at any age examined, the nerve densities of the right ventricle of 30- and 90-day-old SHRSP were significantly greater $(p<0.05)$ than those of age-matched WKY rats (Fig. 5, 6 and 7).

\section{Discussion}

Although many studies have estimated the NA concentration in the heart of SHR of various ages, no definite conclusions have been obtained. While some biochemical studies reported that the NA levels in the heart in SHR and SHRSP were lower
$(10-14)$ or not higher $(15,16)$ than those of WKY rats, others reported that the NA content in the heart in SHR was greater than that in WKY rats (17). Nakamura et al. (18) showed that the NA content in the heart was not significantly different between SHR and WKY rats at 6 weeks of age, while it was lower in SHR than in WKY rats at 12 weeks of age. Watanabe et al. $(19,20)$, however, reported that there was no difference in the NA concentration in the fetal heart between SHR and WKY rats, but the NA concentration was higher in the heart of 16-week-old SHR than in age-matched WKY rats. According to Adams et al. (21), sympathetic activity 


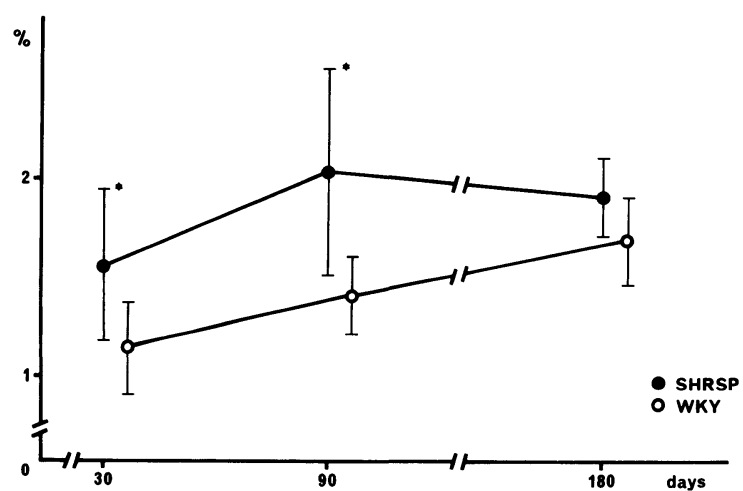

Fig. 5. Comparison of the density of noradrenergic nerve fibers in the myocardium of the right ventricle between $S H R S P$ and $W K Y$ rats at various ages. $* \mathrm{p}<0.05$ compared with WKY rats.

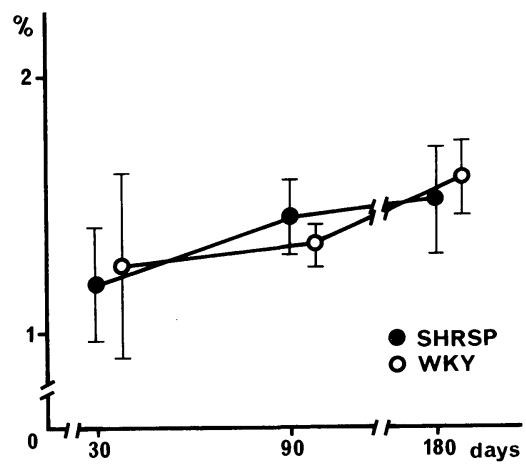

Fig. 6. Comparison of the density of noradrenergic nerve fibers in the myocardium of the left ventricle between SHRSP and WKY rats at various ages.

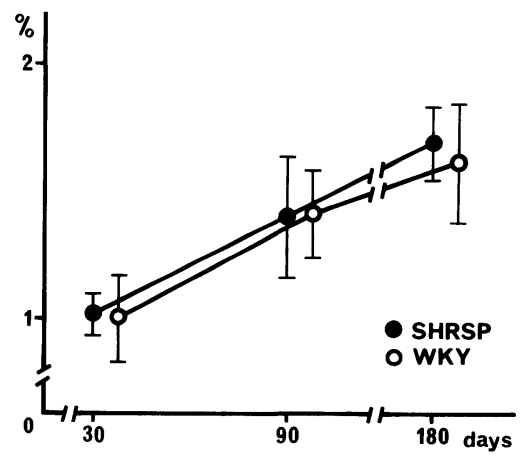

Fig. 7. Comparison of the density of noradrenergic nerve fibers in the myocardium of the ventricular septum between SHRSP and WKY rats at various ages.

in the left ventricle was higher in SHR than in WKY rats through most of the period between 4 and 50 weeks of age.

In the present study, we used a morphological technique to examine and compare the NA nerve fiber densities in the hearts of SHRSP and WKY rats. The densities of NA nerve fibers in the subepicardium of the right and left ventricles were higher in SHRSP than in WKY rats from 10 to 180 days of age, those in the myocardium of the right ventricle were higher in SHRSP than in WKY rats from 30 to 90 days of age, and those in the myocardium of the left ventricle and the ventricular septum in SHRSP were similar to those in WKY rats. These morphometric results suggest that the total amount of NA in the whole heart in SHRSP is larger than that of age-matched WKY rats up to six months after birth.

The hyperinnervation of the heart by NA nerve fibers in SHRSP in this study may be a primary change of the heart prior to hypertension and may be caused by hyperfunction, increased activity of lysine and DOPA uptake, and increased relative weight of the stellate ganglia that innervate the heart (1-3). This is entirely in accord with evidence indicating that perivascular hyperinnervation of the middle cerebral artery and ophthalmic artery in SHRSP results from hyperfunction of the superior cervical ganglia $(4,5)$. Conversely, the present study further supports our previous investigations demonstrating peripheral sympathetic ganglia hyperfunction in SHR and SHRSP (1-5).

The present study demonstrated for the first time regional- and age-related variations in the ratio of NA nerve fiber density in the heart of SHRSP to that of WKY rats. While NA nerve fibers were more densely distributed in the subepicardiums of the right and left ventricles of SHRSP than in those of WKY rats at all ages examined, with the exception of the left ventricle at 90 days of age, myocardial NA nerve fiber densities were higher in SHRSP than WKY rats only in the right ventricle at 30 and 90 days of age. Although the underlying reasons for the regional variations in the ratio of NA nerve fiber density in the heart of SHRSP to that of WKY rats are not known, one reason may be hypertrophy of the heart, especially hypertrophy of the left ventricle, in SHRSP. Cardiac or ventricular hypertrophy has been reported to occur in SHR at 6 and 12 weeks of age (18) or even 1 week after birth (22). The report stating that left ventricle weight/body weight ratios are greater in SHR than in WKY rats at 4,21 and 35 weeks of age, while right ventricle weight/body weight ratios are similar in these strains at 21 and 35 weeks (23) may well account for the regional difference in NA fiber density between SHRSP and WKY rats.

The ratio of NA nerve fiber density in the heart of SHRSP to that of WKY rats was greatest at the youngest ages examined in the epicardium and in the myocardium, respectively, decreasing thereafter in both portions. The reduction in the ratio of NA nerve fiber density between both strains may reflect a decreasing trend in the functional activity of the stellate ganglia with increasing age (1-3).

Increased cardiac NA innervation seems to play an important role in the development and function of the heart in SHRSP, especially in young age because NA has been reported to be necessary for 
proper development of the heart in mouse fetuses (24), while it causes progressive hypertrophy of the heart in young cats $(25)$.

\section{Acknowledgment}

We are grateful to Miss. M. Terada and Mr. K. Okugawa for their technical assistance.

\section{Reference}

1. Kondo M: Autoradiographic study of ${ }^{3} \mathrm{H}$-lysine uptake by superior cervical and stellate ganglia in prehypertensive spontaneously hypertensive rats. Virchows Arch B 1986; 52: 299-304.

2. Kondo M: Autoradiographic study of ${ }^{3} \mathrm{H}$-DOPA uptake by superior cervical and stellate ganglia of spontaneously hypertensive rats during the prehypertensive stage. Virchows Arch B 1987; 54: 190-193.

3. Kondo M, Terada M, Shimizu D, Fujiwara T, Tabei R: Morphometric study of the superior cervical and stellate ganglia of spontaneously hypertensive rats during the prehypertensive stage. Virchows Arch B 1990; 58: 371-376.

4. Kondo M, Miyazaki T, Fujiwara T, Yano A, Tabei $\mathrm{R}$ : Increased density of fluorescent adrenergic fibers around the middle cerebral arteries of stroke-prone spontaneously hypertensive rats. Virchows Arch B 1991; 61: 117-122

5. Tenkova T, Kondo M, Fujiwara T, Tabei R, Tsukahara S: Sympathetic hyperinnervation protects vascular smooth muscle cells from necrosis in stroke-prone spontaneously hypertensive rats. Virchows Arch B 1993; 64: 373-377.

6. Tabei, R, Kondo M, Miyazaki T, Fujiwara T, Terada M, Watanabe Y: Sympathetic hyperinnervation of the coronary artery in the stroke-prone spontaneously hypertensive rat. In Genetic Hypertension, Sassard $\mathrm{J}$ (ed.) Colloque INSERM/John Libbey Eurotext, 1992, Vol 218, pp 241-243.

7. Amenta F, Cavallotti C, De Rossi M, Vatrella F: The autonomic innervation of rat brachial artery and vein. Acta Histochem Cytochem 1983; 16: 472-482.

8. Amanita F, Bronzetti E, Cavallotti C, et al: Quantitative image analysis of the density and pattern of adrenergic innervation of blood vessels of rat spinal cord. J Auton Nerv Syst 1987; 18: 261-264.

9. Kawai Y, Ohhashi T: Histochemical studies of the adrenergic innervation of canine cerebral arteries. $J$ Auton Nerv Syst 1986; 15: 103-108.

10. Johnson EM, Macia RA: Unique resistance to guanethidine-induced chemical sympathectomy of spontaneously hypertensive rats. A resistance overcome by treatment with antibody to nerve growth factor. Circ Res 1979; 45: 243-249.

11. Howes LG, Summers RJ, Louis WJ: The influence of age and sex on cardiac, renal and caudal artery catecholamine content in spontaneously hypertensive
(SHR) and Wistar Kyoto (WKY) rats. J Auton Pharmacol 1986; 6: 171-180.

12. Mano M, Jeffreson S, Head RJ: Vascular, ganglia and cardiac catecholamine disposition in the spontaneously hypertensive rat and in the stroke-prone spontaneously hypertensive rat. J Vasc Res 1992; 29: 8-12.

13. Pahor M, Giudice PL, Bernabei R, et al: Age-related increase in the incidence of ventricular arrhythmias in isolated hearts from spontaneously hypertensive rats. Cardiovasc Drug Ther 1989; 3: 163-169.

14. Wocial B, Chodakowska J, Zukowska-Grojec Z, Rutczynski M, Boratynski W: Tissue catecholamine concentrations in spontaneously hypertensive rats. Acta Physiol Pol 1977; 28: 303-312.

15. Donohue SJ, Stitzel RE, Head RJ: Time course of changes in the norepinephrine content of tissues from spontaneously hypertensive and Wistar Kyoto rats. $J$ Pharmacol Exp Ther 1988; 245: 24-31.

16. Louis WJ, Spector S, Tabei R, Sjoerdsma A: Synthesis and turnover of norepinephrine in the heart of the spontaneously hypertensive rat. Circ Res 1969; 24: 85-91.

17. Head RJ, Cassis LA, Robinson RL, Westfall DP, Stitzel RE: Altered catecholamine contents in vascular and nonvascular tissues in genetically hypertensive rats. Blood Vessels 1985; 22: 196-204.

18. Nakamura $\mathrm{K}$, Gerold M, Thoenen H: Genetically hypertensive rats: relationship between the development of hypertension and the changes in norepinephrine turnover of peripheral and central adrenergic neurons. Arch Pharmakol 1971; 271: $157-169$

19. Watanabe $\mathrm{K}$, Shibata $\mathrm{A}$, Wakabayashi $\mathrm{H}$, et al: Changes in cardiac $\alpha_{1^{-}}$and $\beta_{1}$-adrenergic receptors and $\mathrm{Ca}^{2+}$ binding sites in the myocardium of SHR. Acta Med Biol 1990; 38: 69-74.

20. Watanabe $\mathrm{K}$, Shibata A, Wakabayashi $\mathrm{H}$, et al: Changes in $\alpha_{1^{-}}$and $\beta_{1}$-adrenergic receptors and calcium ion binding sites in the fetal myocardium of spontaneously hypertensive rats (SHR). J Pharmacobiodyn 1991; 14: 182-186.

21. Adams MA, Bobik A, Korner PI: Differential development of vascular and cardiac hypertrophy in genetic hypertension: relation to sympathetic function. Hypertension 1989; 14: 191-202.

22. Weiss L, Lundgren Y: Left ventricular hypertrophy and its reversibility in young spontaneously hypertensive rats. Cardiovasc Res 1978; 12: 635-638.

23. Korner P, Bobik A, Oddie C, Friberg P: Sympathoadrenal system is critical for structural changes in genetic hypertension. Hypertension 1993; 22: 243-252.

24. Thomas SA, Matsumoto AM, Palmiter RD: Noradrenaline is essential for mouse fetal development. Nature 1995; 374: 643-646.

25. Marino TA, Cassidy M, Marino DR, Carson NL, Houser S: Norepinephrine-induced cardiac hypertrophy of the cat heart. Anat Rec 1991; 229: 505-510. 